\title{
A Multi-Wavelet Neural Network Particle Swarm Optimization Based MRPID of Interior Permanent Magnet Synchronous Motor Drives
}

\author{
Aruna Rajamanickam ${ }^{1 *}$, Sairabanu Shaik Mohideen² \\ ${ }^{1}$ Karpagam University, Coimbatore, Tamil Nadu, India \\ ${ }^{2}$ Karpagam University, Coimbatore, Tamil Nadu, India \\ *Corresponding author's Email: raruna0375@gmail.com
}

\begin{abstract}
In this paper, a Multi wavelet neural network (MWNN)-Particle Swarm Optimization (PSO) based MRPID controller is proposed for the speed control of an interior permanent magnet synchronous motor (IPMSM) drive system. In the proposed controller, the multi wavelet transform is used to decompose the available error signal from the actual speed and command speed signal into different frequency components. The parameters of the proposed controller are optimized by PSO algorithm. Then the calculated error coefficients are multiplied with their respective gains to generate an overall control signal. The proposed model is implemented in MATLAB/SIMULINK working platform and the speed control performance of a proposed controller is evaluated. The IPMSM motor drive with MWNN-PID controller through simulation results proves a better performance and stability when compared to that of conventional PID and WNN-PID controllers.
\end{abstract}

Keywords: IPMSM; Wavelet Neural Network; PID controller; Multi resolution Regression PID controller.

\section{Introduction}

Permanent Magnet Synchronous Motors (PMSM) is broadly used in industrial and robotic applications, due to their high competence, low inertia and high torque to volume ratio [1] [2]. It has the benefits of higher competence when compared to induction motors due to the lack of rotor losses and no-load current below the rated speed, its decoupling control performance is much less receptive to the parameter deviations of the motor [3]. As a result, PMSM have found open attention in planning machines for several high performance industrial applications and particularly for hybrid electric vehicle (HEV) and electric vehicle (EV) [4]. In specific, the interior permanent magnet synchronous motors are getting increased attention for high performance drive applications because of their high power density, high competence and flux weakening capability [5]. The magnet of IPMSM is hidden in the rotor core, shows certain good properties such as mechanically robust rotor construction, a rotor physical non saliency, and small efficient air gap [6]. However, the precise speed control of a drive turns out to be a complex issue owing to nonlinear coupling among its winding currents and the rotor speed and the nonlinearity present in the torque equation [7].

The variable speed issues of the motors are usually handled by conventional controllers such as proportional integral (PI), proportional integral derivative (PID) controllers [8], fuzzy neural network controller, adaptive neural network (NN) controller, and wavelet based MRPID controller correspondingly [9]. Although they are uncomplicated, these controllers are very sensitive to parameter differences, change in load, changes in command speed and other uncertainties [10]. The design of PI or PID controller is uncomplicated as the computations of the proportional gain, integral gain and derivative gain are prepared using secondorder method or Ziegler-Nichols method [11]. The uncertainty problem in the PI and PID controller can be accomplished by the fuzzy neural network, in order to develop the speed tracking precision of the conventional controllers, an adaptive and artificial intelligent controllers have been proposed [12].

Recently, wavelet neural networks have been employed in power systems for dissimilar goals, because of their benefits such as the multi-resolution 
of wavelets and the learning of $\mathrm{NN}$ [13]. In the proposed system, the MRPID controllers utilize wavelet theory for the disintegration of the tracking error signals, these controllers have two or more parameters and the number of parameters depends on the level of decomposition [14]. A multiresolution wavelet (MRW) controller is applied for the speed control of traveling wave ultrasonic motor where the wavelet controller is based on wavelet theory and takes benefit of the advanced filtering capability of the wavelet transform [15]. The wavelet transforms have been applied in the modeling, analysis, and control of motor drives for high-performance applications [16]. The wavelet transforms include the capacity to decay wideband signals into time and frequency domains at the same time in order to spotlight on short time intervals for high-frequency components and on long time intervals for low frequency components [17]. The WNN is appropriate for the approximation of unknown nonlinear functions and fast variations [18] [19]. As a result, WNN has been demonstrated to be better than the other neural networks in that the structure can present more potential to enhance the mapping relationship between the inputs and outputs [20]. The paper is organized as follows: section 2 is designated to the problem statement. In section 3, the methodology is discussed in detail. In Section 4, the experimental results are presented and discussed in section, section5 concludes the document.

\section{Recent Research Work: A Brief Review}

Numbers of research works are previously existed in literature which was based on the speed tracking of an IPM motor drive using various optimization techniques. Some of the works are reviewed here Uddin et al. [21] have presented a closed-loop vector control of an interior permanent magnet synchronous motor (IPMSM) drive incorporating two separate fuzzy logic controllers (FLCs). The restrictions of traditional PI controllers were evaded, and the presentation of the drive system was enhanced. While maintaining current and voltage constraints, a flux controller was as well integrated in such a way that both torque and flux of the motor can be powered. Hence, the suggested drive expands the operating speed limits for the motor and facilitates the suitable employ of the reluctance torque. Accordingly the simulation and experimental effects show the better dynamic response in torque and speed for the suggested drive over a broad speed range.

Khan et al. [22] have presented a hybrid neurowavelet scheme for online tuning of a wavelet-based multi resolution proportional integral derivative (MRPID) controller in real time for exact speed control of an interior permanent-magnet synchronous motor (IPMSM) drive system under system uncertainties. In the proposed wavelet-based MRPID controller, the discrete wavelet transform (DWT) was applied to decay the speed error among actual and command speeds into dissimilar frequency components at different scales of the DWT. The MRPID controller parameters were tuned online by the wavelet neural network (WNN) to make definite optimal performance of the drive system. With adaptive learning rates in the closedloop control of the IPMSM drive system the neuro wavelet-based MRPID controller was trained online. The performance of the proposed hybrid controller was examined in both simulation and experiments at dissimilar dynamic operating conditions. The better performance of the proposed WNN-based selftuning MRPID controller was as well authenticated over fixed-gain controllers.

Gilbert Hock Beng et al. [23] have proposed a sensor fault detection and isolation algorithm based on an extended by kalman filters. Frequently, to attain good control performance, the Interior permanent-magnet synchronous motors (IPMSMs), one dc link, and at least two current sensors were used. Breakdown of any of these sensors might lead to humiliate the system performance or even instability. Accordingly, such sensor fault resilient control turns out to be a very significant issue in modern drive systems and it was vigorous to system random noise and competent in real-time execution. In addition, the proposed algorithm was compact and can identify and separate all the sensor faults for IPMSM drives. By widespread experimental results, thorough theoretical analyses were presented and the efficiency of the proposed approach was demonstrated.

Feng et al. [24] have proposed a terminal sliding-mode (TSM) observer for estimating the immeasurable mechanical parameters of permanentmagnet synchronous motors (PMSMs) used for complex mechanical systems. The observer can track the system states in finite time with high steady-state precision. A TSM control strategy was designed to guarantee the global finite-time stability of the observer and, meanwhile, to estimate the mechanical parameters of the PMSM. A secondorder sliding-mode algorithm was designed to soften the switching control signal of the observer. The effect of the equivalent low-pass filter can be properly controlled in the algorithm based on requirements. The smooth signal of the TSM 
observer was directly used for the parameter estimation. The experimental results in a practical $\mathrm{CNC}$ machine tool were provided to demonstrate the effectiveness of the system.

Qiao et al. [25] has proposed a sliding-mode observer (SMO) to achieve the sensor less control of permanent-magnet synchronous motor (PMSM). An observer was built according to the back electromotive force (EMF) model, after the back EMF equivalent signal was obtained. In this way, the low-pass filter and phase compensation module were eliminated, but the estimation accuracy was improved in the observer. Numerical simulations and experiments with a PMSM are carried out. The results demonstrate that the proposed SMO can effectively estimate rotor position and speed and achieve good static and dynamic performance.

Cheng-Kai et al. [26] have proposed a modelfree predictive current control (PCC) of interior permanent-magnet synchronous motor (IPMSM) drive systems based on a current difference detection technique. The model-based on PCC (MBPCC) of IPMSM needs knowledge of parameters such as resistance, q-axis inductance, and extended back EMF, so they proposed a novel model-free approach that improves the need for unnecessary previous knowledge about the system and only exploits the stator currents and the current differences related to different switching states of the inverter. In spite of the significant difference of the suggested approach, it implements a measure similar to that in the MBPCC approach to get the next switching state of the inverter by reducing a cost function. The suggested method was simple to execute due to its plainness and free of any multiplication operation. The experimental effects explain that the suggested method can considerably develop the current-tracking performance of the controller.

Kwon et al. [27] have proposed a design and control method of interior permanent magnet synchronous machines (IPMSMs), which was controlled in sensor less position control mode based on high frequency signal injection, for absolute position estimation and control capability. An IPMSM design with asymmetric winding and asymmetric rotor geometry was suggested and erected as a prototype to understand the mechanical rotor position estimation capability, from a specified template six-pole/nine-slot IPMSM with symmetric structure. The mechanical rotor position can be recognized based on the proposed design at the cost of slight degradation of basic motor features such as torque ripples, harmonics in back EMF, and so on.
The overall presentations of the suggested IPMSM were evaluated thorough finite element method simulations and experiments.

An assessment of the modern investigational works has illustrated the fact that the speed control of an interior permanent magnet synchronous motor (IPMSM) drive under several system ambiguities has become the vital issue in the IPM motor drive mechanism. In order to control the speed of an IPM motor drive system, the $\mathrm{d}$ and $\mathrm{q}$ axis segments of the stator current were employed. The PI and PID controllers have been the initial controllers which were deployed for the speed control of a motor drive system. However, there existed certain issues in as much as these controllers were very susceptible to the parameter change resulting in the issue of chattering. This, in turn, leads to low control precision which downgrades the efficiency of the system ultimately paving the way for instability. In literature the fuzzy logic controllers, terminal sliding-mode (TSM) controller, predictive current controller and an algorithm based on Kalman filter were employed to fine-tune the control efficiency of the system. In this regard, the FLC is found to be highly robust when compared with the PI, PID and sliding mode controllers as the feedback is quick. Moreover there is no overshoot and zero steadystate error but the drive is susceptible to the unanticipated load disturbances. In the terminal sliding mode controller the system tracing faults will converge to zero in an indefinite duration of time. Further the unacceptable chattering of the TSM control system is likely to excite the high frequency feedback of the system. The predictive current controller scales down the computational intricacy. However, the open issue is that only one switching change is likely during a switching period. In literature only limited hybrid technique based works are offered to tackle the menace, these are the deficiencies and issues which have motivated us to perform this investigational work.

In motor drives, absent prior chips on the utilization of wavelet transforms are predominantly hypothetical and depend upon the examination of drive reactions in either reproduction or investigations for low-and medium-speed. Hence, there subsist need to create and actualize a tuning wavelet controller progressively for the IPMSM drive framework. The proposed work presents a MRPID controller based MWNN with PSO for controlling the speed of IPMSM. The benefit of the proposed technique is to utilize the neural system wavelet based MRPID speed controller for elite IPMSM drive frameworks. The intention of utilizing 
the proposed - tuning wavelet-based speed controller in the IPMSM drive frameworks is to conquer the restrictions of settled addition and versatile controller-based IPMSM drive frameworks. The proposed tuning MRPID-controller-based IPMSM drive has effectively executed in Matlab/Simulink platform. The performance of the proposed controller is evaluated through simulation and experimental results.

\section{Modeling of an IPMSM Drive System}

The equivalent circuit model of an Interior permanent magnet synchronous machine in the rotor d-q reference frame [27] is shown in Figure 1.

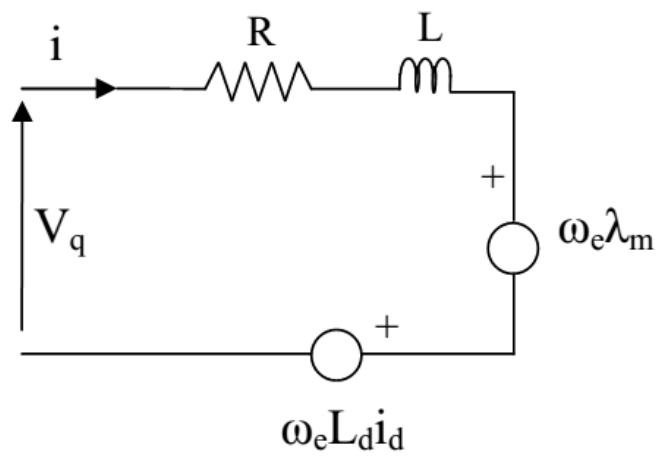

(a)

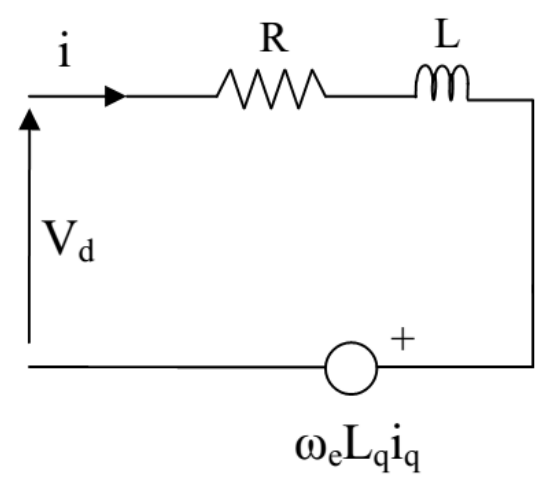

(b)

Figure.1 Equivalent circuit of an IPMSM drive system (a) $q$ axis circuit (b) d axis circuit

The mathematical model of an IPMSM drive can be described by the subsequent equations in a synchronously rotating rotor $\mathrm{d}-\mathrm{q}$ reference frame as [26],

$$
\begin{gathered}
V_{d}=i_{d} R_{s}+\frac{d \lambda_{d}}{d t}-\omega_{e} \lambda_{q} \\
V_{q}=i_{q} R_{s}+\frac{d \lambda_{q}}{d t}-\omega_{e} \lambda_{d} \\
\lambda_{q}=L_{q} i_{q} \\
\lambda_{d}=L_{d} i_{d}+\lambda_{m}
\end{gathered}
$$

The related mathematical model in (1) explains the direct $\mathrm{V}_{\mathrm{d}}$ and quadrature $\mathrm{V}_{\mathrm{q}}$ voltage equations. Make a note of that the motor parameters are not steady but are functions of Id and Iq are not. By substituting the equation (3) and (4) in (1) and (2) the $\mathrm{d}$ and $\mathrm{q}$ axis equations are obtained are shown below.

$$
\begin{gathered}
{\left[\begin{array}{l}
V_{d} \\
V_{q}
\end{array}\right]=\left[\begin{array}{ll}
r_{s}+p L_{d} & -P \omega_{r} L_{q} \\
P \omega_{r} L_{d} & r_{s}+p L_{q}
\end{array}\right]\left[\begin{array}{l}
i_{d} \\
i_{q}
\end{array}\right]+\left[\begin{array}{l}
0 \\
P \omega_{r} \lambda_{m}
\end{array}\right]} \\
T_{e}=\frac{3 p}{2}\left[\lambda_{f} i_{q}+\left(L_{d}-L_{q}\right) i_{d} i_{q}\right] \\
T_{e}=T_{L}+K_{f} p \omega_{r}+B_{m} \omega_{r}
\end{gathered}
$$

The accomplishment of the speed control of an IPMSM drive system is the main intention of this paper. From the equations (5) (6) and (7) it is depicted that the speed control can be attained by controlling the $\mathrm{d}$ and $\mathrm{q}$ axis components of the phase currents. Where id and iq are the direct and quadrature currents (A), $\mathrm{Vd}$ and $\mathrm{Vq}$ are the direct and quadrature voltages (v), Rs is the stator resistance $(\Omega), \mathrm{Ld}$ and $\mathrm{Lq}$ are the direct and quadrature inductances $(\mathrm{H}), \mathrm{T}_{\mathrm{L}}$ is the load torque $(\mathrm{Nm}), \mathrm{Te}$ is the electrical torque $(\mathrm{Nm}), \mathrm{J}$ is the moment of inertia of the motor and load, $\mathrm{K}_{\mathrm{f}}$ is the friction coefficient of the motor, $\omega_{\mathrm{r}}$ is the rotor angular speed $(\mathrm{rad} / \mathrm{s}), \mathrm{p}$ is the number of pole pairs of the motor, $B_{m}$ is the moment of inertia of the motor and load, $\lambda_{\mathrm{m}}$ is the permanent magnet flux (Wb) $\lambda_{\mathrm{d}}$ and $\lambda_{\mathrm{q}}$ are the quadrature and direct flux linkages.

\subsection{Speed control process of proposed hybrid IPMSM drive system}

In this section, MWNN-PSO based speed controller of an IPMSM is described. The motor speed is recognized by monitoring the rotor position using an optical encoder which is mounted on the rotor shaft. By using backward difference interpolation technique, the motor speed and the error value is evaluated. The actual and the command speed difference gives the corresponding speed error values. Then, the error and the change in error values are given as an input to the MWNN. The error signal is processed through the multi wavelet transformation, which is carry out two level decomposition of error signal based on the frequency in terms of low and high frequency error signals. Since there is more information of low and high frequency with multi wavelet decomposition than traditional wavelets, the error gets decomposed to high level by using the multi wavelet transformation. 


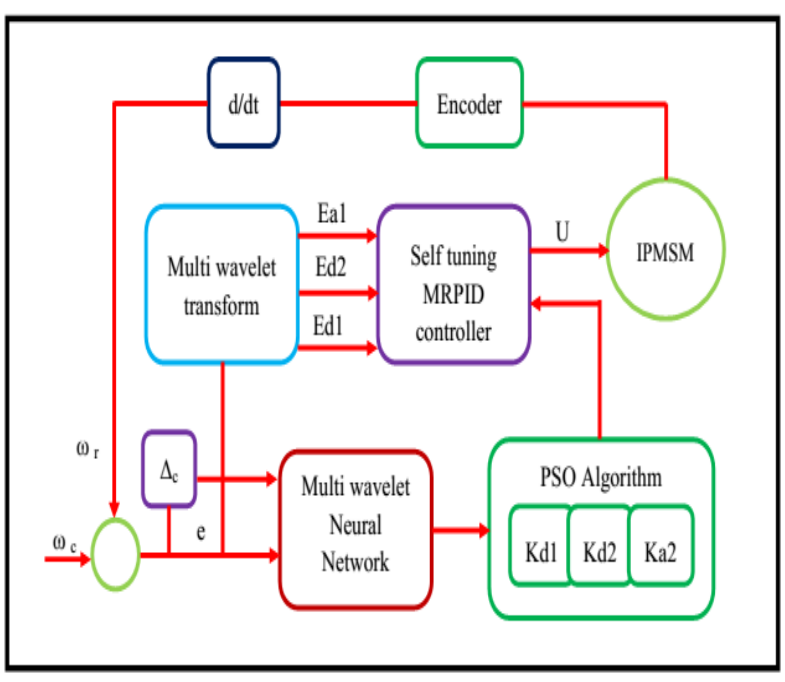

Figure.2 Proposed Multi-wavelet neural network based MRPID controller

The error coefficients gets added along with the gain parameters from the MWNN to produce the efficient control signal u (17) using a self tuning MRPID controller. However, the PSO algorithm is applied to optimize the gain parameters of the Multiwavelet neural network, the gains $K_{d 1}, K_{d 2}$ are used for tuning the high and medium frequency components. The gain $K_{a 2}$ is used for tuning the low frequency components. Thus, the control signal generated by the MRPID controller will control the speed of an IPMSM drive system effectively. Moreover, the controller based on particle swarm optimization (PSO) is used to control the speed of IPMSM motor in wide range and to provide better performance than the conventional WNN based controller. Figure 1 shows the block diagram of the IPMSM motor with MWNN-MRPID controller based on PSO algorithm. The specified explanation of the proposed MRPID controller is described below.

\subsubsection{Description of the Proposed MRPID Controller}

A speculation of PID controller based on multiresolution decomposition of error by utilizing small waves generally known as wavelets. The wavelet decomposition provides much higher resolution than the traditional PID in describing the history and predicting the immediate future of the error. Therefore, this controller is named a multi resolution proportional-integral-derivative (MRPID) controller. The basic idea is that if $\mathrm{e}$ is decomposed as

$$
e=\sum e_{i} \text { where }, i=1,2, \ldots \ldots . . n
$$

Then a generalized PID controller can be formulated as

$$
u=\sum K_{i} e_{i} \text { Where } i=1,2, \ldots . n
$$

Where $\mathrm{K}_{\mathrm{i}}$ are gain parameters to be determined. In general, error and the change in error values are given as an input to the PID controller in order to generate the control output $u$ which is shown in

$$
u_{P i d}=K_{p} e+K_{i} \int e d t+K_{d} \frac{d}{d t} e
$$

The gain value $K_{p}, K_{i}$ and $K_{d}$ are the proportional derivative gains used by the system to act on the error respectively. In terms of frequency information the proportional and integral terms are used to capture the low frequency information of the error signal and the derivative term is used to capture the high frequency information of the error signal. If more resolution in frequency is desired, less resolution in time is achieved by using an MRPID controller, in which the computation time and complexity has also been reduced. An MRPID controller decomposes the error signal into its high, low frequency signals, by applying multi wavelet decomposition to the error signal, the error gets decomposed up to the second level of resolution. Each one of these is scaled by its respective gain and then added together to generate the control signal (u) which is given in (17).

$$
u_{\text {mrpid }}=K_{d 1} e_{d 1}+k_{d 2} e_{d 2}+K_{a 2} e_{a 2}
$$

Based on the level of decomposition of the error signal the MRPID controller can have two or more parameters. If there is a one level decomposition next the error signal is classified as low and high frequency signal and the controller will be containing only two gain parameters. In order to attain high resolution two level decomposition of the error signal is performed, so that there will be a generation of three gain parameters. Each of these gain parameters are inserted together with the error signals to produce the effective control signal.

\subsubsection{Tuning of a MWNN-PSO based MRPID Controller}

\section{Multi -Wavelet Transform}

The wavelet transform is a type of signal transform that is commonly used in compression or for decomposition of the error signal. A newer alternative to wavelet transform is the Multi-wavelet transforms. Multi-wavelets are very similar to wavelets but have some important differences. In particular, Multi-wavelets have two or more scaling and wavelet functions where as wavelets have an 
associated scaling function $\phi(t)$ and wavelet function $\psi(t)$. The set of scaling functions can be written as $\phi(t)=\left[\phi^{1}(t), \phi^{2}(t), \ldots . . \phi^{r}(t)\right]^{T}$, where $\phi(t)$ is called the multi-scaling function. Likewise, the Multi-wavelet function is defined from the set of wavelet functions

as $\psi(t)=\left[\psi_{1}(t) \psi_{2}(t) \ldots \ldots . \psi_{r}(t)\right]^{T}[29]$. Where, $T$ is denoted as the vector response and $\mathrm{r}$ can be arbitrarily large (where $r=2$ ) [30]. The multi wavelet relation of low pass filter and high pass filter is as follows.

$$
\begin{aligned}
& \phi(t)=\sqrt{2} \sum_{k=0}^{n-1} H_{k} \phi(2 t-k) \\
& \psi(t)=\sqrt{2} \sum_{k=0}^{n-1} G_{k} \psi(2 t-k)
\end{aligned}
$$

Where, $H_{k}$ is the low pass filter coefficient and $G_{k}$ is the high pass filter coefficient. The speed error of the IPMSM drive system is applied as an input to the multi wavelet network, so that the error gets decomposed. In multi-wavelet transform, the decomposition produces two low-pass sub-bands, two high-pass sub-bands and two sets of wavelet coefficients for the each level of decomposition. This transformation detects and extracts disturbance features in the form of low and high frequency information. Multi-scaling functions and Multiwavelet functions available in multi-wavelets led to better decomposition of error in each level of decomposition. The extracted features of the error signal help the neural network in distinguishing one disturbance event from another. The detailed description of the Multi wavelet neural network is described in the following section 3.4.2.

\section{Multi Wavelet Neural Network}

The neural network is used to train the proposed controller. In Figure 3 the basic structure of a multi wavelet neural network is shown. The various decomposed error signal for the different motor speed is applied to the neural network. As seen in figure 3 , the inputs of multi wavelet neural network controller are e and $\Delta \mathrm{e}$ where $\mathrm{e}$ is the difference between the actual speed $\omega_{\mathrm{r}}$ and the command speed $\omega_{\text {c. }}$ In order to achieve the efficient speed control of an IPMSM drive system, the neural network should be trained in such a way that for the given input of error $\mathrm{e}$ the controller should produce a proper control signal $u$. The input and output relation of an multi wavelet neural network can be described as

$$
f_{j}=\sum_{t=1}^{r} \sum_{k \in z} c_{j, k} \phi_{j, k}^{t}(t)
$$

The dilation and translation parameters of the multi wavelet neural network is given by

$$
\begin{gathered}
\phi_{j, k}^{l}=2^{j / 2} \phi_{j, k}^{l}\left(2^{j} t-k\right), l=1, \ldots . . r \\
\psi_{j, k}^{l}=2^{j / 2} \psi_{j, k}^{l}\left(2^{j} t-k\right), l=1, \ldots . . r
\end{gathered}
$$

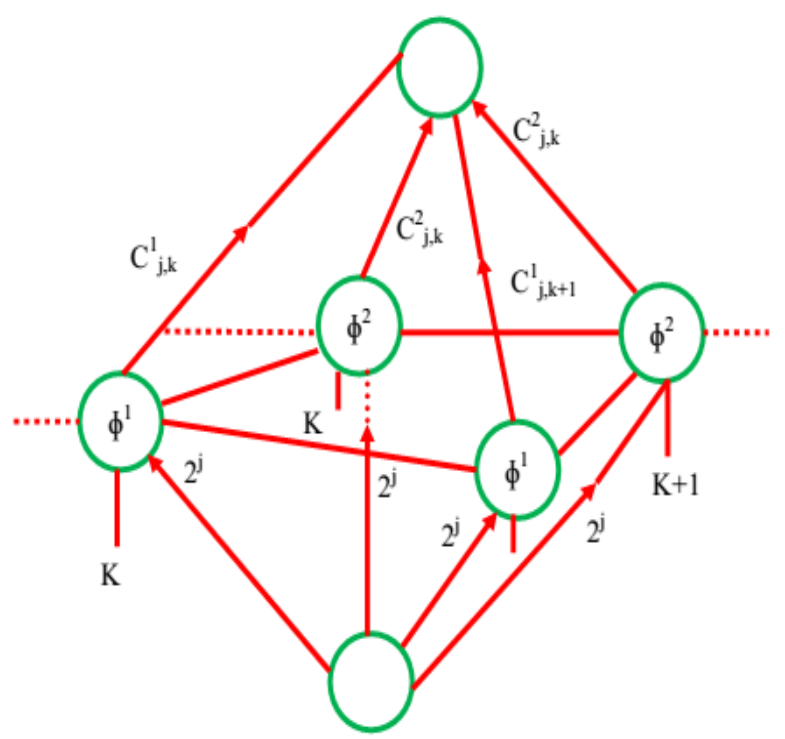

Figure.3 Multi wavelet Neural Network

Where, $2^{\mathrm{j}}$ are the weights of the hidden nodes. The node function in the first row and second row are represented as $\phi^{1}(t)$ and $\phi^{2}(t)$ respectively. The weights of the nodes are $c_{j, k}^{1}, c_{j, k+1}^{1} \ldots \ldots \ldots$ and $c_{j, k}^{2}, c_{j, k+1}^{2} \ldots \ldots . .$. which will be trained in such a way that the hierarchy $\mathrm{j}$ of the multi resolution increases gradually. When the training data set is available, the effective control signal can be obtained by minimizing the error. It is to be noted that there is no input output connection between $\phi^{1}(t)$ and $\phi^{2}(t)$, only segmental lines are used not arrows. The arrows are used only to denote the nodes in the same hidden layer. The weights of the nodes are trained by increasing the hierarchy $\mathrm{j}$ of multi-resolution gradually. The multi wavelet decomposition provides much higher resolution than the traditional PID in describing the history and predicting the immediate future of the error.

\subsubsection{Optimization of Gain Parameters using PSO Algorithm}

In this paper the gain parameters can be optimized by using the PSO algorithm. The particle swarm optimization (PSO) is an evolutionary 
algorithm developed by Kennedy and Eberhart in 1995[29]. The algorithm helps to find an optimal gain parameter values through the interaction of individuals among the various optimal solutions. Initially, the gain parameter values are generated randomly in the search space and each value in the search space represents the optimal solution to the problem. The position and velocity of the gain parameters are represented as X and V respectively. For each and every iteration process the particles will memorize the previous optimal value and the global optimal value in order to update the velocity $\mathrm{V}$. The equation to update the velocity is shown as

$$
\begin{gathered}
V_{i}^{(t+1)}=w^{*} V_{i}^{(t)}+C_{1} r 1^{*}\left(\text { Pbest }_{i}^{t}-x_{i}^{(t)}\right)+ \\
C_{2} r 2 *\left(\text { gbest }_{i}^{t}-x_{i}^{(t)}\right) \\
X_{i}^{(t+1)}=X_{i}^{(u)}+V_{i}^{(t+1)}
\end{gathered}
$$

Where $i=1,2,3 \ldots n$. The term $t$ and $t+1$ stand for the time index of the current and the next iteration respectively. The term $\mathrm{c} 1$ and $\mathrm{c} 2$ are called the acceleration constant which is used to adjust the position of the particles, $\mathrm{r} 1$ and $\mathrm{r} 2$ are the random numbers chosen in between the interval $(0,1), \mathrm{w}$ is the inertia weight factor usually reduces from 0.9 to 0.4 . The inertia weight can be updated by using the formula

$$
w^{(t)}=w_{\max }-t\left(w_{\max }-w_{\min }\right) / \text { iter } r_{\max }
$$

Where $w_{\max }$ and $w_{\text {min }}$ are the maximum and minimum values of $\mathrm{w}$ and iter $_{\max }$ represents the maximum number of iterations. In order to reduce the possibility of particles leaving the search space, the value of each dimension of the velocity is clamped in between the interval $\left[V_{\max }, V_{\min }\right]$. The velocity value is chosen to be $0.1 \leq k \leq 0.5$. The velocity which keeps track of the current speed of the IPMSM drives system. Each value has a current fitness which is obtained by evaluating the error function of the parameters current position. In the optimization process the gains $K_{d 1}, K_{d 2}$ and $K_{a 2}$ are the solutions which is kept constant during the simulation process, the speed errors are added in order to evaluate the candidate solution. To obtain the optimal solution using PSO algorithm, the system model is simulated number of times to search the optimal solution. It is difficult to start the IPMSM drive system continuously. However, it is difficult for each particle of candidate $K_{d 1}, K_{d 2}$ and $K_{a 2}$ parameters to be evaluated within one sampling time, which is usually from $10-100 \mu$ sec. All the gains in the MWNN control system are optimized by minimizing the error in the control signal using the PSO in order to achieve the best speed control performance during the operation.

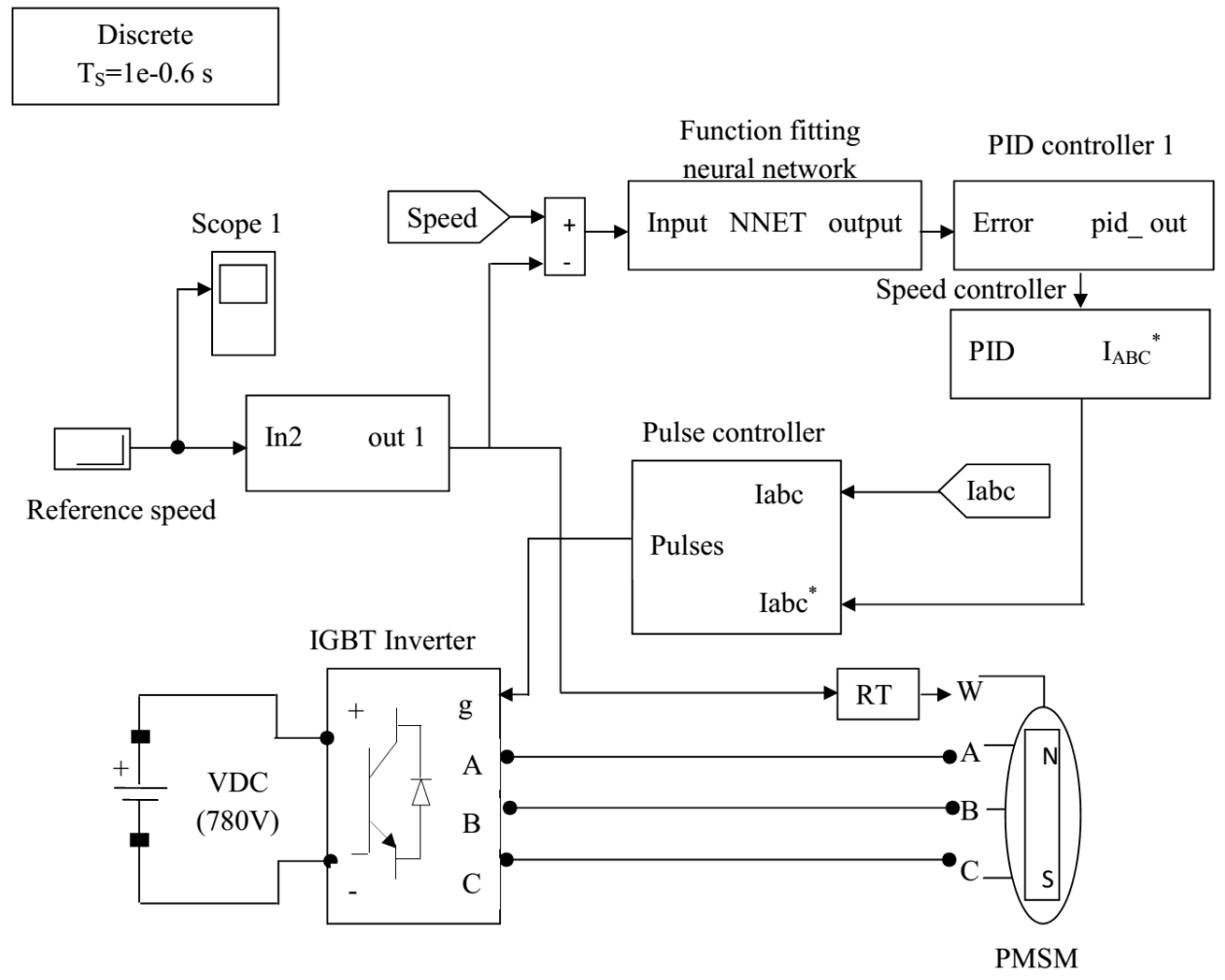

Figure.4 SIMULINK model of the Proposed IPMSM based MRPID controller 


\section{Result and Discussion}

In the paper, the MWNN based MRPID controller is used for the optimal speed control of an IPMSM drive system. The proposed MWNN has two inputs; the speed error and the change of this error which is given to the input layer. The hidden layer has neurons with multi wavelet function. One output in the output layer and the feedback connections for all layers. The translation and dilation factors, weights connection for MWNN and the speed is controlled by tuning the MRPID parameters using PSO algorithm. Figure 4 shows the Simulink model of the proposed speed control strategy. In order to verify the effectiveness of the proposed controller, the performances of the proposed method are evaluated and compared with the existing methods such as, PID and wavelet based MRPID controllers.

\subsection{Performance Analysis of the Proposed and Existing Controller}

In this section, the performance of the IPMSM drives system with MWNN-MRPID controller is determined and illustrated in figure 5, 6 and 7. It shows the speed response of the IPMSM motor for the conventional and the proposed controllers. The actual speed of the motor varies for different controllers with respect to $100 \mathrm{rad} / \mathrm{sec}$ as the reference speed for each $0.01 \mathrm{sec}$. In order to show the step response of the proposed controllers, the reference speed is varied between 100 to $160 \mathrm{rad} / \mathrm{sec}$ and its effectiveness is compared with the conventional PID and MRPID controllers. The speed response of the IPMSM drive system with PID controller was shown in figure (5). In which the reference speed is about $100 \mathrm{rad} / \mathrm{sec}$ and the actual speed of the motor is about $120 \mathrm{rad} / \mathrm{sec}$ at time of about time $\mathrm{t}=0.0053 \mathrm{sec}$. Then after the fraction of second again the speed of the motor reduces to 90 $\mathrm{rad} / \mathrm{sec}$. Then the oscillations in the curve has been reduced and starts to attain the steady state at time $\mathrm{t}=0.01 \mathrm{rad} / \mathrm{sec}$.

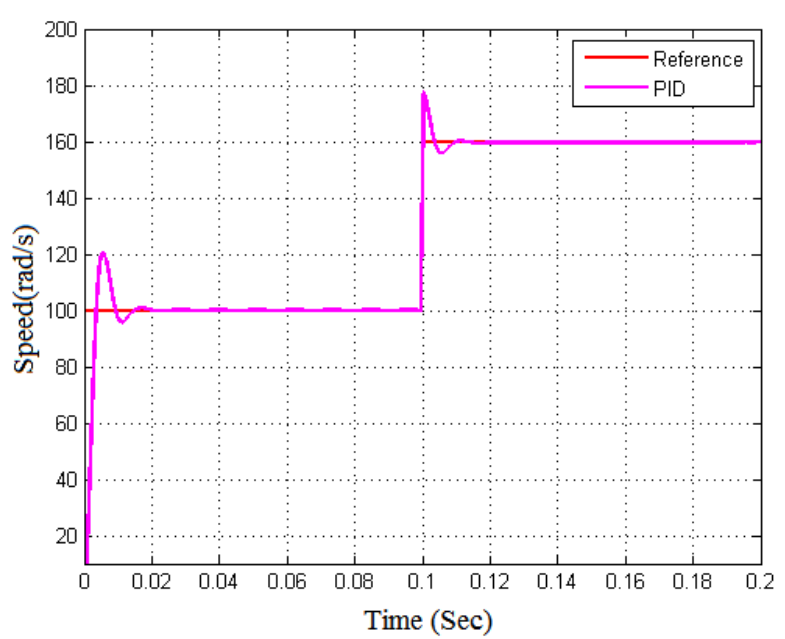

(a)

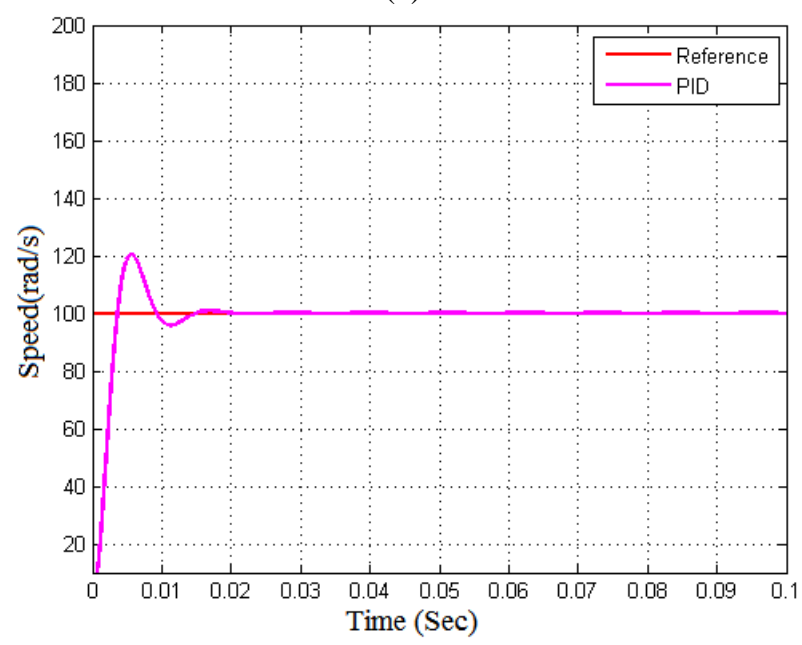

(b)

Figure.5 Speed response of the IPMSM drive system using PID controller

In the wavelet based MRPID controller technique, the speed curve is initially raised at time $\mathrm{t}=0.004 \mathrm{sec}$ and attains the speed of about 115 $\mathrm{rad} / \mathrm{sec}$. At $\mathrm{t}=0.005$, oscillations in the curve has been reduced and then starts to attain the steady state and the speed is of about $100 \mathrm{rad} / \mathrm{sec}$ which is almost equal to the applied command speed. From, the simulated results shown in Fig 6(a)-(b), one can see that the speed responses for the proposed controller is good from the beginning and the differences between the actual speed and the desired command speed is lower than that of the existing PID controller. 


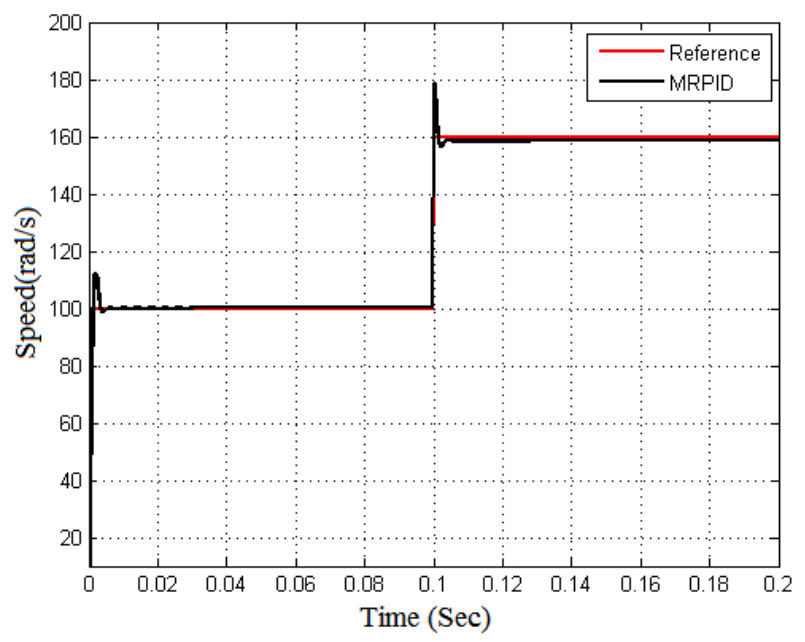

(a)

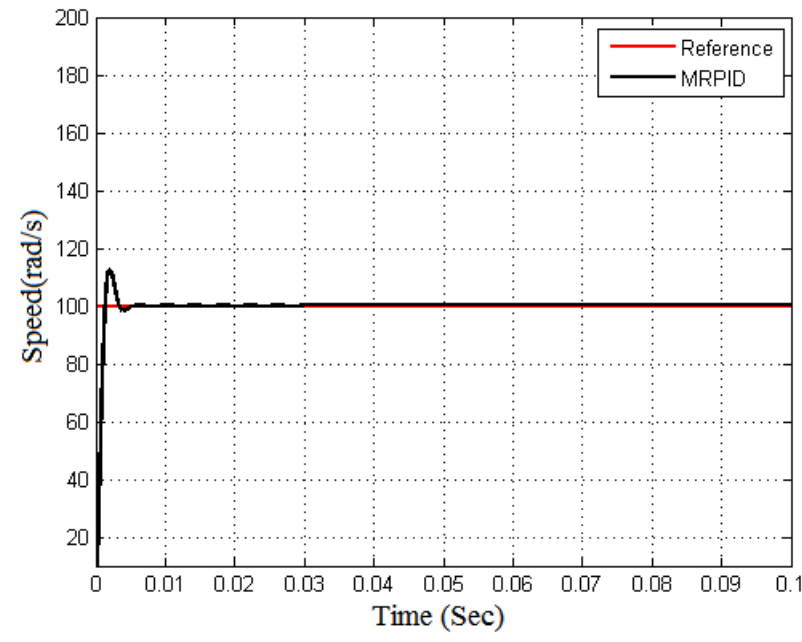

(b)

Figure.6 Performance analysis of speed for the wavelet based MRPID controller

The simulated speed response results of the Proposed MWNN-PSO based MRPID controller for a IPMSM drive system is of about $100 \mathrm{rad} / \mathrm{sec}$ at time $t=0$ is shown in Figure 7 . In this the speed curve is initially raised at time $t=0$ and attains the speed of about $100 \mathrm{rad} / \mathrm{sec}$ and then it goes steadily at the same speed. The reference command speed is varied for the step change of $140 \mathrm{rad} / \mathrm{sec}$ and $160 \mathrm{rad} / \mathrm{sec}$ at time $t=0$ and $t=0.1$ respectively. The output speed response of the IPMSM drive system and the error performances are evaluated using the proposed controller and the conventional controllers is shown in figure (6) and (7). The speed of the IPMSM is analyzed by utilizing the MWNN based controller in [22]. In this paper, an enhanced MRPID controller is used to find out the optimal results of the IPMSM. Generally, the advantage of MRPID controller is mentioned in this section 3 .

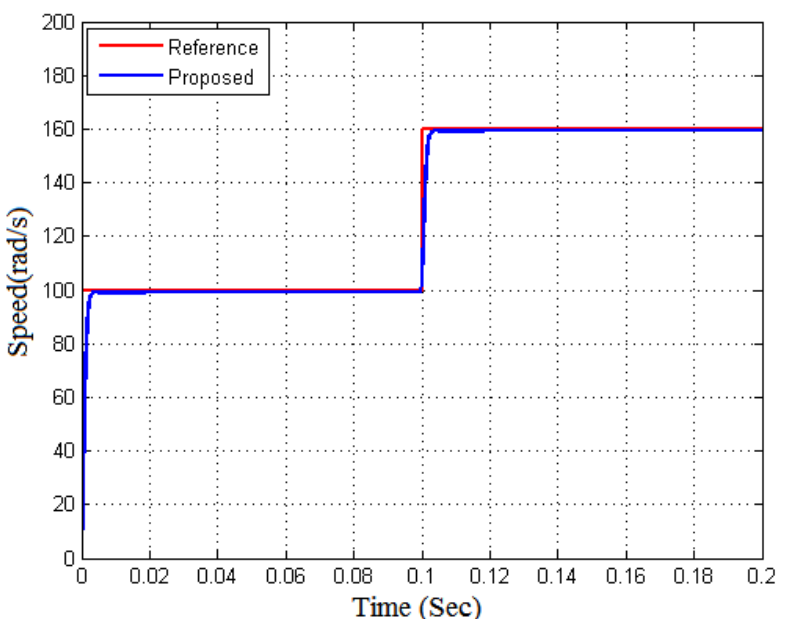

(a)

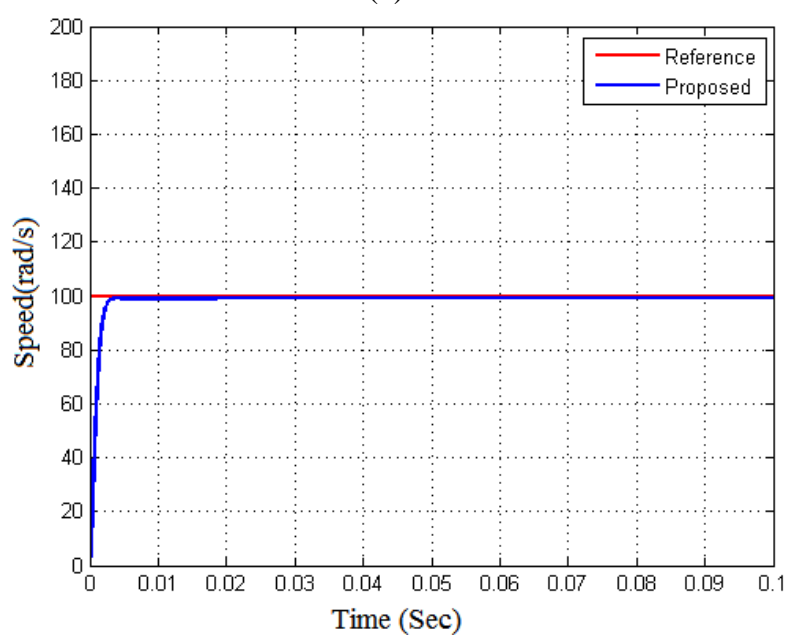

(a)

Figure.7 Performance of the proposed MWNN-PSO based MRPID controller

The compared performance analysis of the proposed MWNN-PSO based MRPID controller with other controllers is depicted in figure 9. The output speed response of the controller for the given reference speed of $100 \mathrm{rad} / \mathrm{sec}$ is shown. It can be seen that while using the bio-inspired particle swarm optimization technique the overshoots obtained is zero as compared to the case when the PID Controller was tuned by using conventional methods. The settling time is also lesser in case of the PSO Optimization, also the rise time is reduced. The MWNN-PSO based MRPID controller tends to approach the reference speed faster and has, comparatively, a zero overshoot. It can be observed from Fig 8 and 9 that the conventional PID controllers have overshoot from the reference speed and attain a steady state with some larger settling time. 


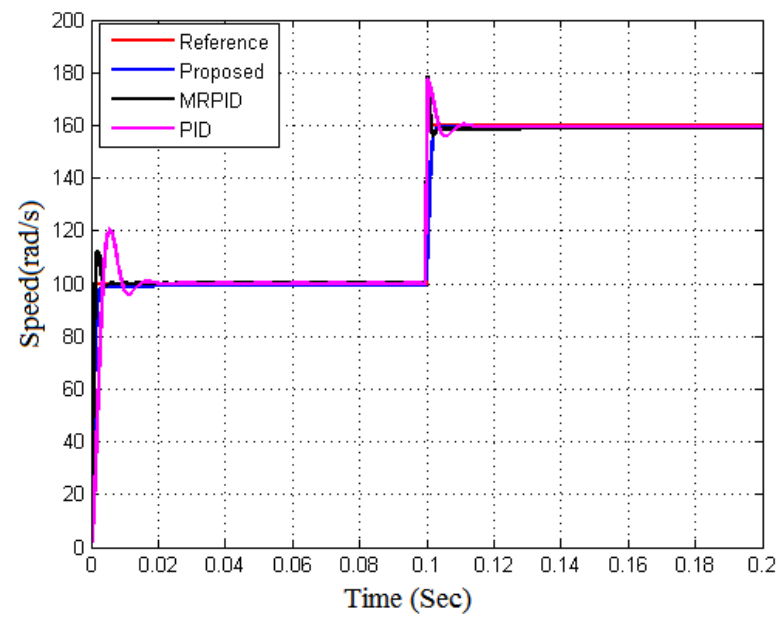

(a)

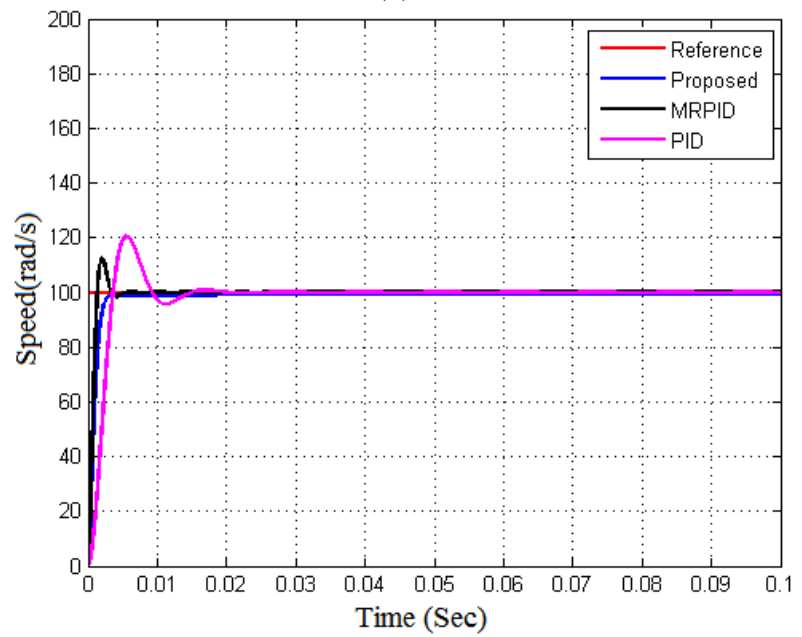

(b)

Figure.8 (a-b) Comparison analysis of the IPMSM drive controllers for step change and for the speed of 100 $\mathrm{rad} / \mathrm{sec}$

At time $\mathrm{t}=0$ the reference speed of $100 \mathrm{v}$ is given as an input, at this situation the speed response of the proposed controller is of about $100 \mathrm{rad} / \mathrm{sec}$ and it will constant up to time $\mathrm{t}=0.1 \mathrm{sec}$ and at this time the reference speed is of about $160 \mathrm{rad} / \mathrm{sec}$ is given as an input. Here, the speed response of the proposed controller. Therefore the comparison analysis of the existing and the proposed illustrates that the proposed controller has zero steady state error and shows $86 \%$ of improvement than that of the existing controller.

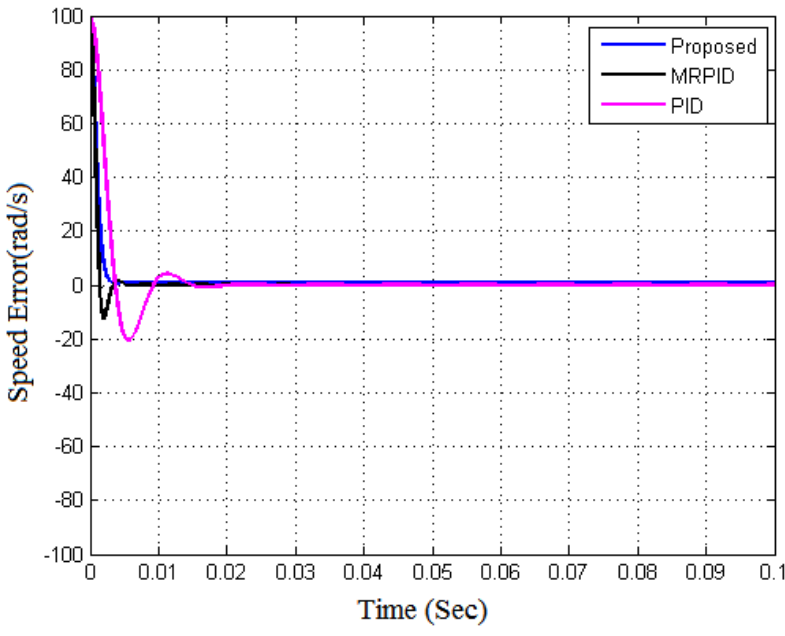

Figure.9 Comparison of Error performance of various controllers

By observing the results at figure (5-10) difference between the actual and the command speed of various controllers gives the corresponding error values which is illustrated in figure (1) and (11). At time $t=0$ the error occurred will be of about $50 \%$ for the existing controller which is highly reduced by using the proposed controller and it attains the steady state at time $\mathrm{t}=0.004 \mathrm{sec}$. The error graph of the proposed controller is shown in figure 10 and the comparison analysis of the controllers is tabulated in table 1 .

Table 1. Comparison in performance

\begin{tabular}{|l|c|r|r|}
\hline Performance & PID & MRPID & Proposed \\
\hline settling time & 0.025 & 0.004 & 0.003 \\
\hline Rise time & 0.0053 & 0.007 & 0.004 \\
\hline Overshoot & 0.0083 & 0.005 & 0.004 \\
\hline
\end{tabular}

Table 1 illustrates a comparison among PID, wavelet based MRPID and the proposed hybrid MRPID speed controller in terms of performance. The comparison includes the calculation of speed rise time, settling time and overshoot. This comparison shows that the proposed method is the best method to overcome the nonlinearity in this drive with high reliability, more robust and good performance than the other methods as can be shown in figure 8 . 


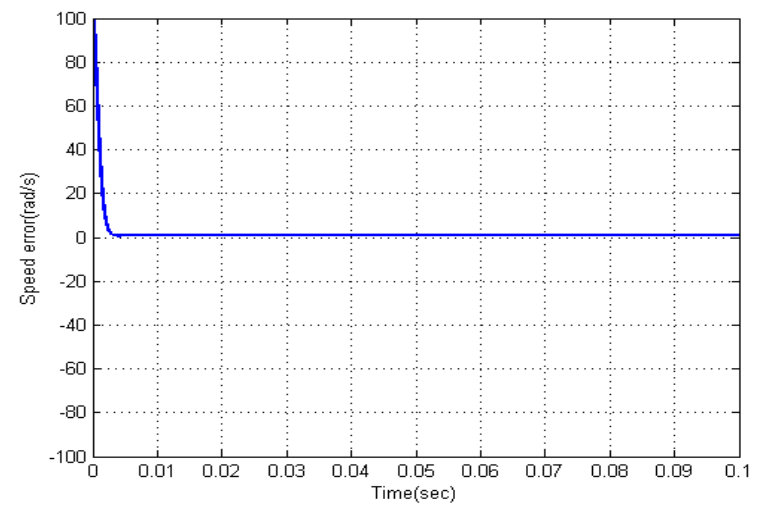

Figure.10 Performance analysis of error using proposed method

From the above illustrations, the performances of the proposed method, with PI, PID and the proposed MWNN based MRPID controller is analyzed by using the speed response and the error obtained by the drive system. From the evaluations, the effectiveness of the proposed method is determined. The effectiveness is evaluated based on the overall performances IPMSM drive system. By using the proposed controller, the IPMSM drive system achieves better speed response with lower error when compared to that of other controllers. The actual speed of the proposed MWNN-PSO based MRPID, wavelet based MRPID and PID controller techniques are evaluated which is of about $100 \mathrm{rad} / \mathrm{sec}, 115 \mathrm{rad} / \mathrm{sec}$ and $110 \mathrm{rad} / \mathrm{sec}$ respectively. From the above analysis the speed control performance of the MWNN based MRPID controller is highly superior to that of the other controller.

\section{Conclusion}

In this paper, an optimal MWNN-PSO based MRPID controller is designed for an optimal speed control of an interior permanent magnet synchronous motor. The multi wavelet decomposition, which represents the error signal at different scales, provides higher resolution representation of the error signals than the one used in existing PID and is the basis of the new controller design. A generalized MRPID controller based on the MWNN is proposed, which is similar to the conventional PID controller, but it is intuitive and effective. The controller gains have an explicit relationship with the characteristics of the error signal, which makes tuning of the controller insightful using PSO algorithm. The Multi wavelet decomposition, which represents the error signal at different scales, provides higher resolution representation of the error signal than the one used in the existing PID and is the basis of the new controller design. Implementation of the Multi wavelet neural network based multi resolution controller in SIMULINK shows promising results, particularly in its ability to provide smooth control effort and better disturbance rejection compared to that of the existing PID controller was analyzed and their results are demonstrated. In future, the performances of speed and torque control will be analyzed by utilizing the advanced PID controller. Moreover, the recent optimization technique will be utilized to find out the optimal control results.

\section{References}

[1] E. Shahat, Adel, and H. E. Shewy, "Permanent Magnet Synchronous Motor Drive System for Mechatronics Applications", International Journal of Research and Reviews in Applied Sciences, Vol. 4 No. 2, pp. 323-338, 2010.

[2] M. M. Kayalvizhi and M. Akilandeswari, "Design and Implementation of Speed Regulator for a PMSM Using Genetic Algorithm, "International Journal of Innovative Research in Science, Engineering and Technology, Vol. 3, No. 1, pp. 866-872, 2014.

[3] Yaou, Zhang, Z. Wansheng and K. Xiaoming, "Control of the permanent magnet synchronous motor using model reference dynamic inversion", WSEAS Transactions on Systems and Control, Vol. 5, pp. 301-311, 2010.

[4] H. Dogan, F. Wurtz, A. Foggia and L. Garbuio, "Performance Analysis and Comparison of Three IPMSM with High Homopolar Inductance for Electric Vehicle Applications", In proceeding of IEEE conference on Power Electronics, pp. 1-10, 2011.

[5] J. Rezaie, M. Gholami, R. Firouzi, T. Alizadeh, K. Salashoor, "Interior Permanent Magnet Synchronous Motor (IPMSM) Adaptive Genetic Parameter Estimation ", In Proceedings of the World Conference on Engineering and Computer Science, pp. 1-5, 2007.

[6] M. Abdesh, S. K. Khan and M. A. Rahman, "An adaptive self-tuned wavelet controller for IPM motor drives", Power and Energy Society General MeetingConversion and Delivery of Electrical Energy in the 21st Century, pp. 1-4, 2008.

[7] A. Rahman, M. D. Vilathgamuwa, M. N. Uddin, and K. J. Tseng, "Nonlinear control of interior permanent-magnet synchronous motor", IEEE Transactions on Industry Applications, Vol. 39, No. 2, pp. 408-416, 2003.

[8] N. Wahid, and N. Hassan, "Self-tuning fuzzy pid controller design for aircraft pitch control", IEEE International Conference on Intelligent Systems, Modelling and Simulation (ISMS), pp. 19-24, 2012.

[9] M. A. S. K. Khan, G. H. George and M. A. Rahman, "Intelligent speed controllers for IPM motor drives", 
In proceeding of Canadian Conference on Electrical and Computer Engineering, pp. 833-836, 2009.

[10]S. R. Vaishnav, and Z. J. Khan, "Design and Performance of PID and Fuzzy Logic Controller with Smaller Rule Set for Higher Order System", In Proceedings of the World Conference on Engineering and Computer Science, pp. 24-26, 2007.

[11] Nour, Mutasim, O. Bouketir and E. Yong, "Selftuning of PI speed controller gains using fuzzy logic controller", Modern Applied Science, Vol. 2, No. 6, pp. 55-65, 2008.

[12] M. A. Khan and M. N. Uddin, "A Novel Wavelet Neural Network Based Robust Control of an Interior Permanent Magnet Motor Drives", IEEE Transaction on Industrial application, pp. 1-8, 2011.

[13] T. T. Lee, C. F. Hsu, and K. N. Hung, "DC-DC Intelligent Power Regulator Design Using Wavelet Neural Network", In Proceedings of IEEE International Conference Networking, Sensing and Control, pp. 751-756, 2007.

[14] Jeevan, G. Leena and V. Malik, "A wavelet based multi-resolution controller", Journal of Emerging Trends in Computing and Information Sciences, Vol. 2, pp. 17-21, 2010.

[15] Sant, A. Vilas and K. R. Rajagopal, "PM synchronous motor drive with wavelet controller and fractional order integrator", In proceeding of Joint International Conference on Power Electronics, Drives and Energy Systems (PEDES), pp. 1-8, 2010.

[16] J. L. F. Daya, V. Subbiah, A. Iqbal, and S. K. Padmanaban, "Novel Wavelet-Fuzzy Based Indirect Field Oriented Control of Induction Motor Drives", Journal of Power Electronics, Vol. 13, No. 4, 2013.

[17]M. A. S. K. Khan, and M. A. Rahman, "Implementation of a new wavelet controller for interior permanent-magnet motor drives", IEEE Transactions on Industry Application, Vol. 44, No. 6, pp. 1957-1965, 2008.

[18]Z. Barton, "Robust control in a multimachine power system using adaptive neuro-fuzzy stabilisers", In proceeding of IEEE conference on Generation, Transmission and Distribution, Vol. 151, No.2, pp. 261-267, 2004.

[19] Farahani and Mohsen, "Design of an Intelligent Controller Based on Wavelet Neural Network to Improve the Stability of Power Systems", Artificial Intelligence and Applications, Vol. 1, No. 1, pp. 1-20, 2014.

[20]F. F. M. E. Sousy, "Robust adaptive Hœ position control via a wavelet-neural-network for a DSPbased permanent-magnet synchronous motor servo drive system", IET Electric Power Applications, Vol. 4, No. 5, pp. 333-347, 2010.

[21]N. Uddin Mohammad and R. S. Rebeiro, "Performance Analysis of an FLC Based Online Adaptation of Both Hysteresis and PI Controllers for IPMSM Drive", IEEE Transaction on Industry Applications, Vol. 48, No. 1, pp. 1-8, 2010.
[22] Khan, M. A. S. K and M. A. Rahman, "A novel neuro-wavelet-based self-tuned wavelet controller for IPM motor drives", IEEE Transactions on Industry Applications, Vol. 46, No. 3, pp. 1194-1203, 2010.

[23] G. H. Beng, Foo, X. Zhang and D. M. Vilathgamuwa, "A sensor fault detection and isolation method in interior permanent-magnet synchronous motor drives based on an extended Kalman filter", IEEE Transactions on Industrial Electronics, Vol. 60, No. 8, pp. 3485-3495, 2013.

[24]Feng, Yong, X. Yu, and F. Han, "High-order terminal sliding-mode observer for parameter estimation of a permanent-magnet synchronous motor", IEEE Transactions on Industrial Electronics, Vol. 60, No. 10, pp. 4272-4280, 2013.

[25] Qiao, Zhaowei, T. Shi, Y. Wang, Y. Yan, C. Xia, and $\mathrm{X}$. He, "New sliding-mode observer for position sensor less control of permanent-magnet synchronous motor", IEEE Transactions on Industrial Electronics, Vol. 60, No. 2, pp. 710-719, 2013.

[26] C. Kai, Lin, T. H. Liu, L. C. Fu and C. F. Hsiao, "Model-free predictive current control for interior permanent-magnet synchronous motor drives based on current difference detection technique", IEEE Transactions on Industrial Electronics, Vol. 61, No. 2, pp. 667-681, 2014.

[27] Y. C. Kwon, S. K. Sul, N. A. Baloch, S. Murakami and S. Morimoto, "Design and Control of IPMSM Sensor less Drive for Mechanical Rotor Position Estimation Capability", IEEE Journal of Emerging and Selected Topics in Power Electronics, Vol. 2, No. 2, pp. 152-158, 2014.

[28] M. Abdesh, S. K. Khan and M. A. Rahman, "An adaptive self-tuned wavelet controller for IPM motor drives", IEEE Power and Energy Society General Meeting-Conversion and Delivery of Electrical Energy in the 21st Century, pp. 1-4, 2008.

[29] Sabra, Maha, B. Khasawneh and M. A. Zohdy, "Nonlinear Control of Interior PMSM Using Control Lyapunov Functions", Journal of Power and Energy Engineering, Vol. 2, No. 1, pp. 17-26, 2014.

[30] L. Jiao, J. Pan and Y. Fang, "Multi wavelet Neural Network and Its Approximation Properties", IEEE Transactions on Neural Networks, Vol. 12, No. 5, pp. 1060-1066, 2001.

[31] Shi, Yuhui and R. Eberhart, "A modified particle swarm optimizer", In proceeding of on IEEE International Conference on Computational Intelligence, pp. 69-73, 1998.

[32] R. krishnan, Sudhakar and J. Subramaniam, "Novel Image Compression Using Multi wavelets with SPECK Algorithm", Int. Arab J. Inf. Technology, Vol. 5, No. 1, pp. 45-51, 2008

[33] Jeevan, G. Leena and V. Malik, "A wavelet based multi-resolution controller", Journal of Emerging Trends in Computing and Information Sciences, Vol. 2, pp. 17-21, 2010 